\title{
An Efficient Gateway Election Algorithm for Clusters in MANET
}

\author{
Sakshi Joshi \\ Research Scholar \\ Department of CSE \\ MITM, Indore
}

\author{
Rakesh Verma \\ Assistant Professor \\ Department of CSE \\ MITM, Indore
}

\author{
Anil Singh \\ Associate Professor \\ Department of CSE \\ MIST, Indore
}

\begin{abstract}
The dynamic nature of Mobile ad hoc network brought several constraints like scalability, robust connectivity, limited power constraints etc. Several cluster based routing algorithms have been proposed for large scale ad hoc networks. In cluster-based routing, the network is logically divided into groups called clusters. The aim of this grouping is to sustain a steady and effective network topology. The election of gateways during inter cluster routing is a critical issue. In this paper, an optimized gateway election algorithm, have been proposed which aims at minimizing the control overhead required in the set up and maintenance of the network, while maintaining robust connectivity. This technique is a unique way of addressing the issues of dynamic adaptation through a hierarchical cluster based approach along with Virtual Identifier. The simulation results show that the proposed technique gives better performance over various gateway election algorithms in terms of packet delivery ratio, routing overhead, end to end delay, and throughput.
\end{abstract}

\section{General Terms}

Clustering, gateway election

\section{Keywords}

MANET, NS2, Cluster, Node.

\section{INTRODUCTION}

Clustering is a means of grouping nodes using some approach to forward data effectively. In clustering, a node called cluster head is chosen within each cluster. The task of cluster head is to maintain a set of nodes that belong to the same cluster as cluster head. These nodes are called cluster members. Cluster head keeps a route to every cluster member. Proactive methods are adopted to update these routes. In addition, a cluster head also keeps a set of gateways belonging to the neighboring clusters.

Gateway election is a crucial step in optimizing the performance of any clustering algorithm. After cluster formation there is two types of routing in MANET. The first one is intra-cluster routing in which both source and destination lie in the same cluster. The second is inter-cluster routing in which source and destination both lies in different cluster. In this case we need the help of gateways. When cluster client is registered with more than one cluster head that it can be assigned the role of gateway. A cluster client work as gateway in inter-cluster routing. When a cluster head needs to communicate with another cluster head it does so via gateway. The cluster head sends the packet to gateways and gateway forward it to the destination cluster head.
Now it is possible that during inter cluster routing there may be some gateways with same length of virtual identifiers then we need to elect one gateway among them. In case if no election is performed and the packets are forwarded to all the gateways with same virtual identifier it will unnecessarily increase network traffic as there will be many redundant copies of packets in the network.

For the sake of clarity, the problem mentioned above is explained diagrammatically.

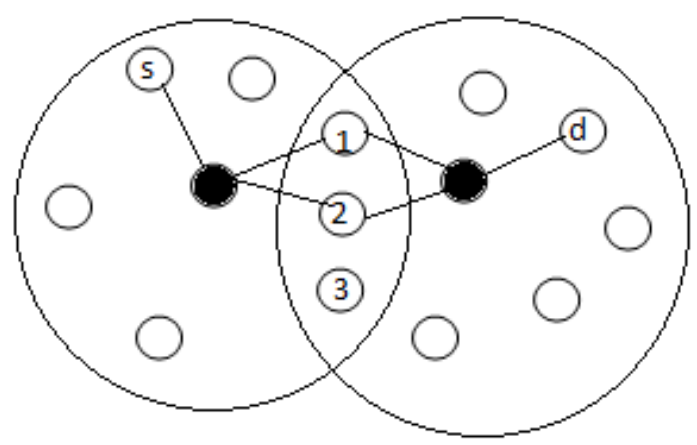

Fig. 1 Inter cluster packet transmission

As it can be seen in Fig. 1, when an ordinary node in the cluster has some data to be delivered it sends it to the cluster head. Now it is the responsibility of the cluster head to find the path to the destination. The cluster head has two routes available from source node to destination node. One path is via node one and the other are via node two. Now if the cluster head forwards the packet through both the gateways there will be multiple redundant copies of the packets in the network. This will in turn also consume a lot of bandwidth which is a scarce resource.

\section{LITERATURE SURVEY}

Rekha Basavraju, et al [1] proposed a minimal gateway election approach to strengthen connectivity in MANET. In MANET enabling efficient communication among different domains is a basic networking problem and one of the areas of research topics. Gateways are to be selected to support connectivity of the nodes present in different domains. Due to mobility of nodes gateway assignment has to be done dynamically. Only a subset of nodes qualifies to become gateway nodes but we cannot use all of them simultaneously. Because they would generate a lot of network overhead since all the gateway nodes may forward the packet and hence there will be a number of redundant copies in the network. In this paper minimum number of gateways is selected by 
considering the neighbor distribution, geographical distance, minimum hops and least load path.

Ben Alla, et al [2] proposed a new protocol for cluster head and gateway election in wireless sensor network by making use of clustering. It makes use of different fuzzy parameters for cluster head and gateway election. In order to elect cluster head two fuzzy parameters are used. These are efficiency and cluster distance. The efficiency is obtained by taking the ratio of residual energy of each node and average energy of cluster. Cluster distance is the summation of distance between the node and the other nodes which are within the cluster. Gateway election is performed on the basis of nodes energy and their proximity to base station.

You $\mathrm{Lu}$, et al [3] proposed a gateway election algorithm for inter domain routing in MANET. It describes I-GIDR (Improved geographical inter domain routing protocol). Paper describes an algorithm for gateway election that makes use of neighbor distribution and neighbor number. Another parameter that it makes use of is neighbor priority. Its a concept based on the geographical distribution and density of neighbor.

MANET is a collection of mobile nodes. Mobility is an inherent feature of this type of networks. In cluster based network, we know that huge responsibility lies on cluster head and gateway for broadcasting packet. So if the selected cluster head is highly mobile it has to be re-elected frequently. Therefore Pal S, Singh S.P [4] proposed a cluster head and gateway selection algorithm based on mobility. The distinct dynamic character of MANET cannot be reflected as most of the clustering schemes assume low mobility. The algorithm considers the mobility characteristics of a node as a metric for gateway calculation. Clustering has several advantages. One is increased resource utilization, because nodes within the clusters can communicate with each other without affecting the nodes in other clusters. It optimally manages network topology by dividing the task among cluster head and gateways.

Cognitive radio network are developed on top of licensed network. Cognitive radio is also known as secondary user. Secondary user should not obstruct the primary users and use the vacant portion of the band. Thus, depending on channel availability, secondary users form cluster to deal with the issue of assignment of channel. Najam ul Hasan et al [5] proposed a mechanism to select gateway nodes to improve inter cluster connectivity. Gateway node is selected by cluster head for adjacent cluster. Gateway nodes are selected considering into account the degree of connectivity of nodes to strengthen the connectivity

Seung Hoon Lee [6] proposes a scheme for election of gateways in which many attributes of the border nodes have been considered for selecting them as gateways. Only a subset of nodes qualifies to become gateway nodes but we cannot use all of them simultaneously. Because they would generate a lot of network overhead since all the gateway nodes may forward the packet and hence there will be a number of redundant copies in the network .In this paper four parameters are considered for gateway election. They are network connectivity, Secure routing, Resource balancing, on demand gateway election.

Deepak Kumar Patel, et al [7] proposed a gateway discovery algorithm for ad hoc network. The algorithm proposed author is adaptive. The author focuses on improving the performance of ad hoc network when it integrates with internet. A number of challenges are faced during this integration. A bridge between MANET and internet is gateway. The algorithm enhances the quality of service of transmitted data and lowers the overhead due to congestion. Intermediate nodes in the network maintain a table which is used by the gateway election algorithm. Whenever a node has some data to deliver to the internet gateway selection algorithm is followed.

\section{CLUSTER FORMATION METHOD}

Clustering is a means of grouping nodes using some approach to forward data effectively. There are various methods in literature for determination of cluster head in MANET. Some of the approaches are-

- $\quad$ Lowest ID Algorithm

- Highest Degree Algorithm

- $\quad$ Node Weight Algorithm

- Weighted Clustering Algorithm

In the proposed work method, highest degree algorithm is used in order to elect cluster head.

\subsection{Hierarchical Cluster Based Approach}

Initially when a node enters a system and desires to join the cluster it sends a request to the cluster head seeking permission to join the cluster. Cluster head records the new node into its member list and sends back acceptance message. Thus, the cluster head is known to every node.

Whenever a node ' $\mathrm{s}$ ' desires to transmit packet to some node ' $d$ ' then route discovery mechanism is carried out. There are two possibilities. Firstly, the source and destination node may be in the same cluster. In this case the route is readily available with the cluster head. Secondly, source and destination may belong to separate clusters. In this case cluster head flood the network using the cluster head and gateways.

The proposed methodology of gateway election reduces this flooding to great extent. This can be well understood by the addressing scheme used in the proposed work and the concept of virtual identifiers as explained in the next section.

\subsection{Proposed Addressing Scheme}

Initially when a node enters a system each node is given some specific ID. The proposed clustering schemes makes use of virtual identifiers (VID) which are expressed as

$$
\mathbf{x . y}
$$

where,

$\mathrm{x}$ being the cluster head ID and $\mathrm{y}$ being the node ID.

Let us consider the same with Fig. 2. A node with ID 2 is the cluster head. Now nodes $1,4,5,9$ are its cluster clients with virtual identifiers $2.1,2.3,2.2,2.4$.

There is another cluster with cluster head having node ID 6 . Its cluster members are 3, 8, 7, 9 with virtual ID 6.1, 6.2, 6.4, 6.3 


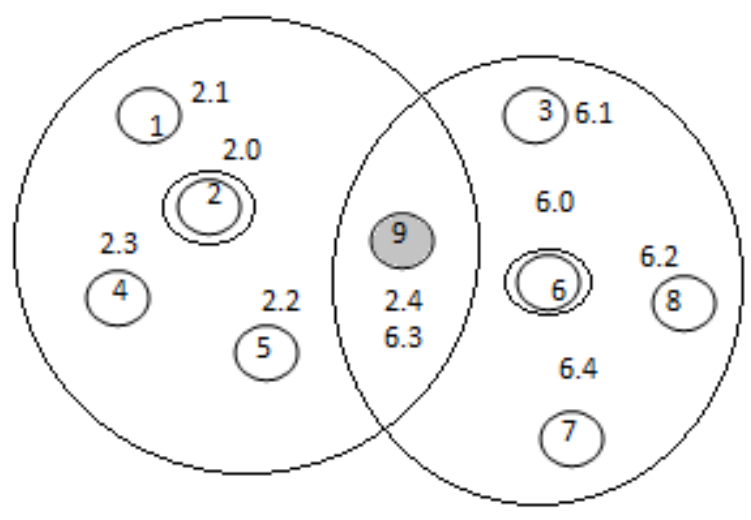

Fig.2 Addressing scheme

During route discovery source cluster head maps the destination address with the list of available gateway nodes. If there is a single gateway between source and destination cluster head, than packets are forwarded through it. After mapping if the ' $x$ ' field of the virtual identifier of the destination matches to more than one gateways than gateway election needs to be performed.

\section{GATEWAY ELECTION METHOD}

In this section we propose a gateway election algorithm based on parameters like transmission range, remaining battery power and mobility.

\subsection{Stages of a node in cluster formation}

Any normal node entering into the network may be in different states after cluster formation. A normal node may be elected a cluster head or if not elected as a cluster head it may join the cluster head and become cluster client. Now, if a cluster client joins more than one cluster head than its chosen as gateway node. Different states of a node can be seen $n$ Fig.3. There are four possible states in which a node can be:

NORMAL NODE: An unassigned node which can either become cluster head, ordinary node or gateway.

CLUSTER HEAD: It is the normal node elected as cluster head.

CLUSTER CLIENT: Node joining the cluster head.

GATEWAY: A cluster client joining more than one cluster.

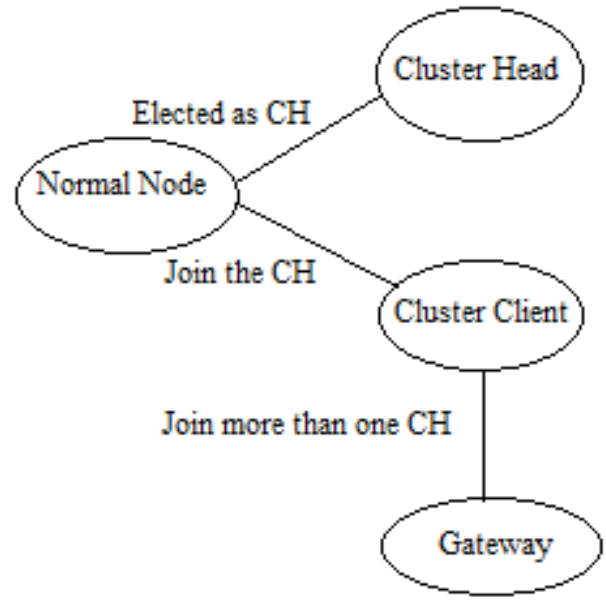

Fig.3 States of a Node

\subsection{Algorithm}

The proposed algorithm is used for election of gateway nodes in clusters. The algorithm follows below mentioned steps:

Step 1: Start

Step 2: Determine the number of gateways between source and destination cluster head

Step 3: If single gateway

a) No need to perform election

Else

b) Go to step 4

Step 4: For each gateway node between source and destination cluster head do

a) Calculate remaining energy $P_{e}[$ node $]$

b) Calculate mobility $\mathrm{P}_{\mathrm{m}}$ [node $]$

c) Calculate transmission $P_{t}[$ node $]$

Step 5: End for

Step 6: Initialize

$\max =\mathrm{P}_{\mathrm{t}}[0], \max 1=\mathrm{P}_{\mathrm{e}}[0], \min =\mathrm{P}_{\mathrm{m}}[0]$

Step 7: For all 'i' gateway nodes do

$$
\begin{aligned}
& \text { If }\left(P_{t}[i]>\max \right) \\
& \text { Max }=P_{t}[i], A=i \\
& \text { If }\left(P_{e}[i]>\max 1\right) \\
& \text { Max }=P_{e}[i], B=i \\
& \text { If }\left(P_{m}[i]<\min \right) \\
& \text { Min }=P_{m}[i], C=i
\end{aligned}
$$


Step 8: end for

Step 9: if $(A=B \& \& B=C)$

Gateway $=\mathrm{i}$

Else

Go to step 7

Step 10: Stop

The proposed algorithm for gateway election is executed after cluster formation. In this algorithm the first step is to determine the count of gateways common among the source and the destination cluster. Now it is a possibility that there may either be a single gateway or multiple gateways. In case there is a single gateway available for inter cluster communication than there is no option left but to forward the packet through that gateway. If multiple gateways are there then in order to reduce flooding, a single gateway among those available gateways is elected. For this election parameters such as transmission range, mobility and remaining battery power are considered. Each of these parameters is computed for the available gateways and then the comparison is performed among them. The gateway node with maximum value of transmission range, minimum value of mobility and maximum value of remaining battery power is elected and then the inter cluster packet transmission is performed using the selected gateway node.

\section{Explanation of the notations}

In the above algorithm $\mathrm{P}_{t}$ is the transmission range of the node, $\mathrm{P}_{\mathrm{e}}$ is the remaining energy of the node. $\mathrm{P}_{\mathrm{m}}$ is the mobility of node; it is taken by computing the running time average speed of every node' n' during a specified time T.

\section{SIMULATION \& RESULTS}

To evaluate the performance of the proposed gateway election method, the algorithm has been simulated in NS-2. The simulation parameters considered for the purpose are as mentioned in the table below.

Table 1. Simulation Parameters

\begin{tabular}{|l|l|}
\hline \multicolumn{1}{|c|}{ Parameter } & \multicolumn{1}{c|}{ Value } \\
\hline Routing protocol & AODV \\
\hline Simulation dimension & 1000 X 1000 \\
\hline Initial energy in joules & 100 \\
\hline Simulation Time & 20 seconds \\
\hline Traffic & CBR \\
\hline Channel Type & Wireless channel \\
\hline No. of nodes & 20 \\
\hline Queue size & 50 \\
\hline Packet Size & 512 bytes \\
\hline
\end{tabular}

Performance Metrics:

Packet Delivery Fraction: The amount of packets successfully delivered at the target network device is known as the packet delivery ratio.

$$
\mathrm{PDF}=(\text { Prcv/Psent }) * 100
$$

Where Prcv is total Packet received \& Psent is the total Packet sent.

Fig.4 shows the comparative PDR of the proposed and existing approach. In this diagram the $\mathrm{X}$ axis shows the number of nodes and the $\mathrm{Y}$ axis shows the packet delivery ratio in terms of percentage. According to the comparative outcomes of the system the proposed approach delivers more packets as compared to the existing approach. The packet delivery ratio of both approaches decreases with the increase in number of nodes.

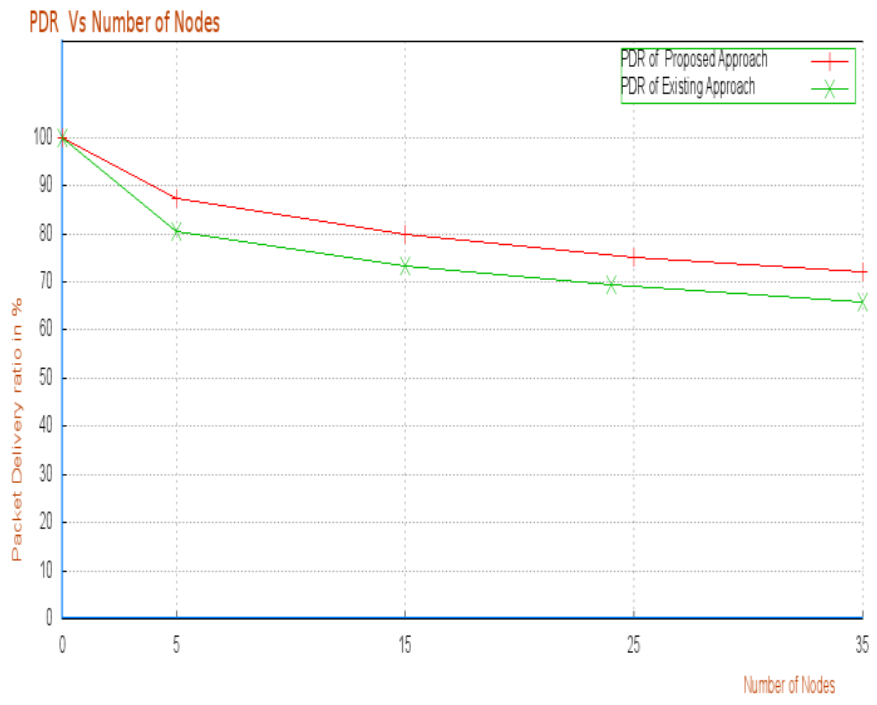

Fig.4 Comparative PDR

Throughput: Network throughput is the degree of message delivered successfully in a communication medium. The data may go through physical or logical connections, or by using a specific network device. The throughput is basically calculated in terms of bits per second. Occasionally it is also calculated in terms of number of data packets per time slot or data packets per second.

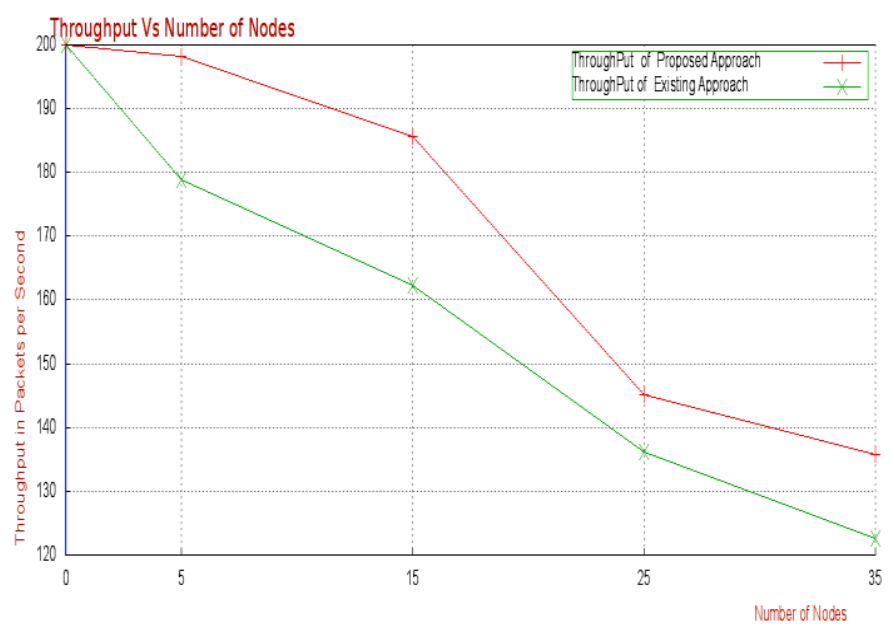

Fig.5 Comparative Throughput

Comparative throughput of the existing and proposed approach is given using Fig.5. In this diagram the $\mathrm{Y}$ axis demonstrates the obtained throughput in terms of Packets per second and the $\mathrm{X}$ axis shows the number of nodes. According to the obtained results the performance of the proposed 
approach in terms of throughput is adoptable due to higher throughput as compared to the existing approach.

End to End Delay: It can be defined as the total amount of time required to successfully deliver a data packet to a targeted network device is known as the end to end delay.

$$
\mathrm{E} 2 \mathrm{E}=(\operatorname{Trcv}-\mathrm{Tsent})
$$

Where Trcv is receive Time and Tsent is sent Time.

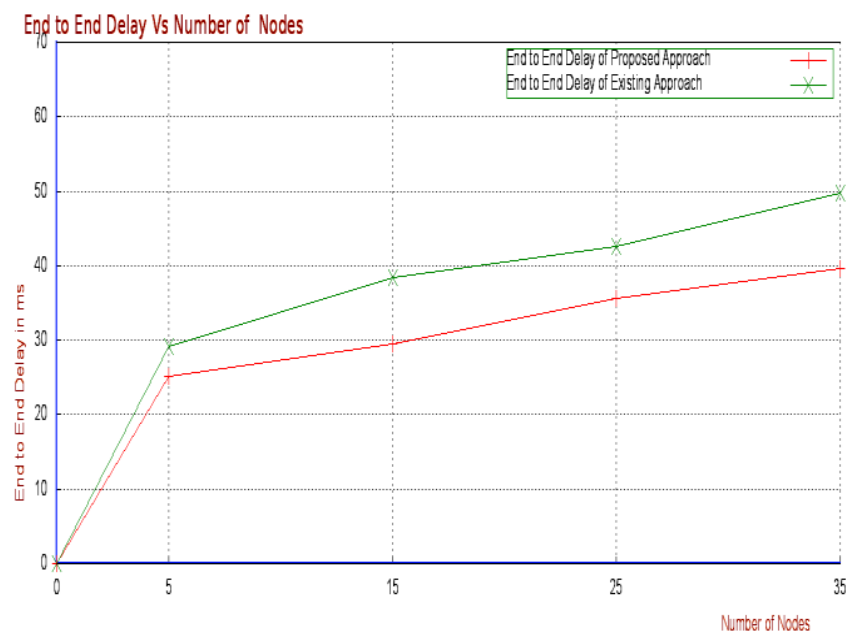

Fig.6 Comparative End to End Delays

According to the obtained end to end delay of the proposed approach is fewer than the existing Approach. Comparative demonstration is provided using Fig.6. As given in figure 5.3 the performance of the proposed approach is adoptable due to less amount of end to end delay as compared to the existing approach.

Routing Overhead: It is associated with the control information sent over the network. Nodes often change their position in the network so there are some old routes present in the routing table that leads to unnecessary routing overhead.

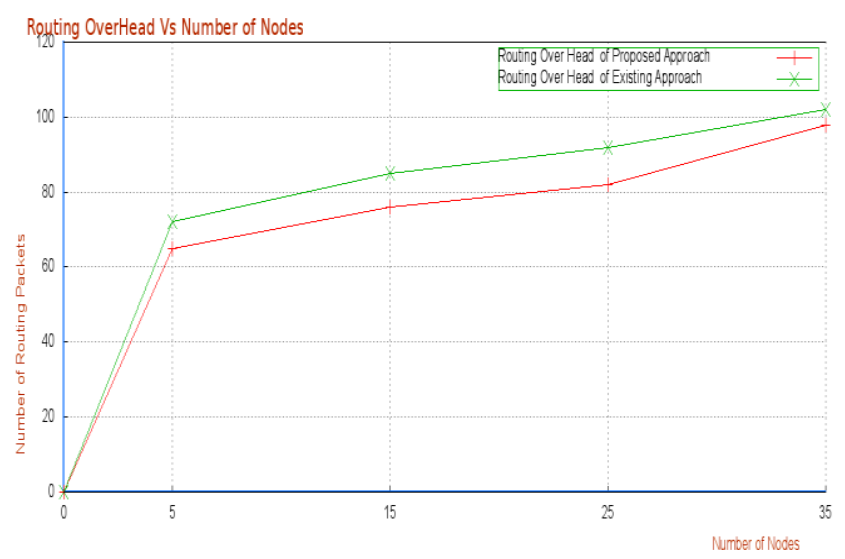

Fig.7 Comparative Routing Overhead

According to the obtained results the routing overhead of the existing approach is higher than the proposed approach. High routing overhead demonstrate the low performance of the system, thus the performance of proposed approach is more efficient than the existing one.

\section{CONCLUSION}

The proposed work focuses on electing a single gateway node among the set of available gateways so as to avoid unnecessary redundant transmission of packet during inter cluster communication. Election of gateways is done by using parameters such as transmission range, mobility and remaining battery power. All these parameters contribute to an efficient gateway election. Also the excessive flooding that takes place clustering during inter cluster packet transmission due to redundant forwarding of packets by many gateways gets reduced. The gateway node with maximum value of transmission range, remaining battery power and minimum value of mobility is chosen for transmission. The work is evaluated using AODV routing protocol. When compared with the existing approach, a significant increase in performance is observed. The performance summary is given below-

a. Packet Delivery Ratio of the proposed approach is higher than that of traditional approach.

b. The proposed approach exceeds in performance to the traditional approach in terms of throughput.

c. Routing overhead of proposed approach is less than existing approach.

d. The proposed approach is more efficient in terms of end to delay.

\section{REFERENCES}

[1] Rekha Basavaraju and D.V. Ashoka "An enhanced geographical based minimal gateway selection method to improve connectivity in MANETs", in Proceedings of International Conference on Emerging Research in Computing Information, communication and application, ERCICA, Elsevier journal, Bangalore,2013, pp. 8-13

[2] Ben Alla, S., Ezzati, A. and Mohsen, A. "Gateway and Cluster head election using fuzzy logic in heterogeneous wireless sensor network", in International Conference Multimedia computing and systems(ICMS) IEEE, Tangier, 2012, pp. 761-766.

[3] You Lu, Biao Zhou, Ian $\mathrm{Ku}$ and Mario Gerla, "Connectivity Improvement for Inter-Domain Routing in MANETs", in Military Communications Conference MILCOM, IEEE, San Jose CA, 2010, pp. 617- 622.

[4] Sapna Pal, S.P Singh, "Mobility based cluster head and gateway selection algorithm in MANET", International Journal of Engineering Research and Technology, vol. 2, Issue 1, 2013, pp. 1-7.

[5] Najam ul Hasan, Waleed Ejaz, Kamran Manzoor, Hyung Seok Kim" GSM: Gateway Selection Mechanism for Strengthening Inter-Cluster Coordination in Cognitive Radio Ad hoc Network" EURASIP Journal on Wireless Communication and Networking, 2013

[6] Seung-Hoon Lee, Nishanth Alapati, Kang-Won-Lee, Mario Gerla, "Multiple Metric Gateway Election in Heterogeneous MANETs", in Army Science Conference, U.K., 2010

[7] Deepak Kumar Patel, Rakesh Kumar "Performance Evaluation of an Adaptive Gateway Discovery Algorithm for Mobile Ad hoc Networks ", International Journal of Computer Science and Information Technologies, vol. 4 , Issue 3, 2013, pp. 436-441 